\title{
MEASUREMENTS OF UNSTEADY WAKE INTERFERENCE BETWEEN TANDEM CYLINDERS
}

\author{
Luther N. Jenkins ${ }^{*}$, Dan H. Neuhart ${ }^{*}$, Catherine B. McGinley ${ }^{*}$, Meelan M. Choudhari ${ }^{\dagger}$, and Mehdi R. Khorrami ${ }^{\dagger}$ \\ NASA Langley Research Center, Hampton, Virginia, 23681
}

A multi-phase, experimental study in the Basic Aerodynamics Research Tunnel at the NASA Langley Research Center has provided new insight into the unsteady flow interaction around cylinders in tandem arrangement. Phase 1 of the study characterized the mean and unsteady near-field flow around two cylinders of equal diameter using 2-D Particle Image Velocimetry (PIV) and hot-wire anemometry. These measurements were performed at a Reynolds number of $1.66 \times 10^{5}$, based on cylinder diameter, and spacing-to-diameter ratios, L/D, of 1.435 and 3.7. The current phase, Phase 2, augments this dataset by characterizing the surface flow on the same configurations using steady and unsteady pressure measurements and surface flow visualization. Transition strips were applied to the front cylinder during both phases to produce a turbulent boundary layer upstream of the flow separation. For these flow conditions and L/D ratios, surface pressures on both the front and rear cylinders show the effects of $L / D$ on flow symmetry, pressure recovery, and the location of flow separation and attachment. Mean streamlines and instantaneous vorticity obtained from the PIV data are used to explain the flow structure in the gap and near-wake regions and its relationship to the unsteady surface pressures. The combination of off-body and surface measurements provides a comprehensive dataset to develop and validate computational techniques for predicting the unsteady flow field at higher Reynolds numbers.

$\begin{array}{ll}D & =\text { cylinder diameter } \\ C_{d} & =\text { drag coefficient, per unit span } \\ C_{P} & =\text { pressure coefficient, } \frac{p-p_{\infty}}{q_{\infty}} \\ C_{P}, & =\text { fluctuating pressure coefficient, } \frac{\sigma_{P}}{q_{\infty}} \\ L & =\text { distance between cylinder centers } \\ p & =\text { pressure measured on model surface } \\ p_{\infty} & =\text { freestream static pressure } \\ q_{\infty} & =\text { freestream dynamic pressure } \\ S t & =\text { Strouhal Number, } \frac{f D}{U_{\infty}} \\ U_{\infty} & =\text { freestream velocity } \\ X & =\text { streamwise coordinate } \\ Y & =\text { vertical coordinate } \\ \mathrm{Z} & =\text { spanwise coordinate }\end{array}$

\footnotetext{
${ }^{*}$ Research Engineer, Flow Physics and Control Branch

${ }^{\dagger}$ Research Engineer, Computational Aerosciences Branch, Associate Fellow, AIAA
} 


$\begin{array}{ll}\Delta Z & =\text { distance between transducer and reference transducer for spanwise pressure correlations } \\ \theta & =\text { azimuthal position } \\ \sigma_{P} & =\text { pressure standard deviation } \\ \omega_{Z} & =\text { spanwise vorticity component } \frac{\partial V}{\partial X}-\frac{\partial U}{\partial Y}\end{array}$

\section{Introduction}

For the past several years, researchers at NASA have been performing experimental and computational studies on tandem cylinder arrangements to better understand the noise generation process and develop accurate noise prediction methods for landing gear systems. The tandem cylinder arrangement produces the type of unsteady flow interaction commonly associated with landing gear systems where upstream components, directly exposed to the oncoming flow, act as bluff bodies and generate unsteady wakes that impinge on one or more downstream components. As such, it provides a canonical problem to advance modeling techniques for the prediction and analysis of unsteady flow interactions.

Numerous studies have been conducted on the tandem configuration due to its practical application in systems used for cooling, venting, and structural support. In most cases, the studies were performed at subcritical Reynolds numbers (less than $1.5 \times 10^{5}$ ) and focused on fundamental issues such as boundary layer development, numerical prediction of flow interference, flow-induced vibrations, and characterization of the wake structure for various cylinder spacings. ${ }^{1-4}$ In Reference 5, Zdravkovich characterizes flow patterns associated with various separation distances (L/D) between the cylinders. For $\mathrm{L} / \mathrm{D}<1.1$, the two cylinders behave as a single bluff body with vortex shedding occurring at the rear cylinder only. For $1.1<\mathrm{L} / \mathrm{D}<1.6$, the shear layers from the front cylinder attach alternately to the rear cylinder and vortex shedding occurs on the rear cylinder. For $1.6<\mathrm{L} / \mathrm{D}<2.5$, the shear layers from the front cylinder attach to the rear cylinder and vortex shedding occurs on the rear cylinder. For separation distances, $2.5<\mathrm{L} / \mathrm{D}<3.2$, intermittent shedding can be detected in the region between the cylinders and vortex shedding occurs on the rear cylinder. For $3.2<\mathrm{L} / \mathrm{D}<3.8$, the flow between the cylinders is bistable and switches between intermittent shedding and constant shedding. For L/D $>3.8$, vortex shedding occurs on both cylinders with the same characteristics as a single cylinder. Quantitative details about the time-averaged and instantaneous flow structure for several of the flow regimes described above have been obtained by Lin, et al. ${ }^{6}$ at $\operatorname{Re}=1.0 \times 10^{4}$ using Particle Image Velocimetry (PIV).

For supercritical Reynolds numbers, $\mathrm{Gu}^{7}$ has shown from static pressure measurements that two flow regimes can exist for tandem cylinders depending on whether the rear cylinder is located in or out of the wake cavity of the front cylinder. For $\mathrm{L} / \mathrm{D}<2.8$, the rear cylinder is located in the wake cavity and the shear layers from the front cylinder either envelope or impinge directly on the rear cylinder. For $\mathrm{L} / \mathrm{D} \geq 2.8$, the rear cylinder is out of the wake cavity and the shear layers from the front cylinder do not impinge directly on the rear cylinder. These descriptions provide useful information about the time-average flow between the cylinders but quantitative information about the unsteady flow field is also needed to enable computer model assessment and validation at critical and post-critical Reynolds numbers. Most studies of the unsteady flow field between tandem cylinders have used fluctuating surface pressure measurements and flow field spectra obtained using hot-wire anemometry to examine the effects of Reynolds number, freestream turbulence, surface roughness, and cylinder spacing on the flow structure associated with the different flow regimes described above. ${ }^{8-11}$ Recent studies by Jenkins, et al. ${ }^{12}$, Khorrami, et al. ${ }^{13}$, and Henderson and Brooks ${ }^{14}$ have added to this body of knowledge by providing additional insight into the unsteady near-field flow structure and its implications on noise radiation.

The work by Jenkins, et al. ${ }^{12}$ and Khorrami, et al. ${ }^{13}$ constitute Phase 1 of a multi-phase study to measure and characterize the unsteady flow on two cylinders in tandem arrangement at a post-critical Reynolds number. The purpose of the current phase, Phase 2, is to document the steady and unsteady surface flow. The results from the current phase, along with those from Phase 1, provide a comprehensive dataset for 1) characterizing the interaction of the wake of the front cylinder with the rear cylinder at two different L/D's, 2) examining differences between the wake of the entire tandem configuration and a single cylinder due to this interaction and 3) developing and validating computational techniques for the prediction of unsteady flows. 


\section{Experimental Apparatus and Techniques}

\section{A. Test Facility}

The experiment was conducted in the NASA-Langley Basic Aerodynamics Research Tunnel (BART). This tunnel is a subsonic, atmospheric wind tunnel used to investigate the fundamental characteristics of complex flow fields and to acquire detailed data for the development and validation of computational fluid dynamics (CFD) models and methods. The tunnel has a closed test section with a height of 28 inches $(0.711 \mathrm{~m})$, a width of 40 inches $(1.016 \mathrm{~m})$, and a length of 120 inches $(3.048 \mathrm{~m})$. The free stream velocity was set to $184 \mathrm{ft} / \mathrm{s}(56 \mathrm{~m} / \mathrm{s})$ during Phase 1 and $144 \mathrm{ft} / \mathrm{s}(44 \mathrm{~m} / \mathrm{s})$ during Phase 2 to achieve a Reynolds number based on cylinder diameter of $1.66 \times 10^{5}$. At these conditions the free stream turbulence level is less than $0.10 \%$. Additional information about the BART can be found in References 15-17.

\section{B. Model}

The tandem arrangement used for both phases of this study consisted of two circular cylinders which spanned the entire height of the test section. Figure 1 shows an installation photo and Fig. 2 provides a schematic of the models with the coordinate reference system. For Phase 1, each cylinder measured 1.75 inches $(44.45 \mathrm{~mm})$ in diameter and 27.188 inches $(690.6 \mathrm{~mm})$ in length and was attached to a base that was inserted into a track mechanism secured to the tunnel floor. With this arrangement, the front cylinder was fixed and the rear cylinder could be translated upstream and downstream to achieve the desired spacing. In addition, data on a single cylinder could be acquired by fixing the location of the front cylinder and removing the rear cylinder. Each cylinder was instrumented with lower, center, and upper static pressure rings located at 10 inches, 14 inches, and 18 inches $(254.0 \mathrm{~mm}, 355.6 \mathrm{~mm}$, and $457.2 \mathrm{~mm}$ ), respectively, from the tunnel floor. The upper and lower rings consisted of 18 orifices spaced every 20 degrees around the cylinder circumference, whereas the center ring consists of 36 orifices spaced every 10 degrees around the cylinder circumference. In addition, 9 pressure orifices with 2 inch $(50.8 \mathrm{~mm})$ spacing were installed along the span of each cylinder at $\theta=180^{\circ}$ to monitor pressure recovery and check for spanwise variations.

For Phase 2, the same arrangement of static pressure orifices was used but in addition, 22 piezoresistive, differential pressure transducers were installed in each cylinder. To accommodate the pressure tubing and electrical wiring, two new cylinders measuring 2.25 inches $(57.15 \mathrm{~mm})$ in diameter were manufactured for use with the same track mechanism used in Phase 1. Increasing the diameter of the model increased the tunnel blockage to 5.63\% compared to $4.37 \%$, the blockage for Phase 1 . The transducers were mounted inside the cylinders in two arrays: a spanwise array of 15 transducers along the length of the cylinder at $\theta=135^{\circ}$ and a circumferential array of eight transducers at the center of the cylinder evenly spaced at $45^{\circ}$ and including the transducer from the longitudinal array at $\theta=135^{\circ}$. To minimize flow disturbance, each transducer was mounted beneath the surface of the cylinder under a pinhole of approximately 0.05 inches $(1.270 \mathrm{~mm})$ in length and 0.02 inches $(0.508 \mathrm{~mm})$ in diameter. The fabrication process for installing the transducers in each cylinder left a conical chamber between the transducer face and the pinhole leading to the surface. This geometrical configuration raised concern about data contamination due to a Helmholtz-type resonance ${ }^{18}$. Calculations indicated that this type of resonance would likely occur around 14 $\mathrm{kHz}$ and subsequent checks on a sample configuration in the NASA Langley Flow Impedance Test Facility confirmed that the resonance frequency was higher than the frequency range of interest for this experiment.

To produce a turbulent boundary layer and achieve the type of flow separation associated with post-critical Reynolds numbers, transition strips were attached along the entire span of the upstream cylinder in the regions between $\theta=50^{\circ}$ and $\theta=60^{\circ}$ and between $\theta=300^{\circ}$ and $\theta=310^{\circ}$. The transition strip was made of "pinked" tape with a nominal thickness of $0.229 \mathrm{~mm}$. "Pinked" denotes that one edge of the tape was "straight" while the other had a "saw-tooth" pattern. The strip was applied with the "straight" edge facing upstream and the "pinked" edge facing downstream. In Fig. 3, the effectiveness of the transition strip in producing a post-critical type flow is evident by the good agreement between mean $C_{P}$ distributions for a single cylinder from Phases 1 and 2 and data from Roshko ${ }^{19}$ acquired at a Reynolds Number of $8.6 \times 10^{6}$.

\section{Measurement Techniques}

The measurement techniques used in this study were selected based on their ability to meet the primary objective of each phase. In Phase 1, 2-D Particle Image Velocimetry provided detailed insight into the steady and unsteady near-field flow structure. The process used to acquire and process the PIV data is described in Ref. 12. Since the objective of Phase 2 was to document and characterize the flow on the surface of each cylinder, steady and unsteady 
pressures were measured on the front and rear cylinders simultaneously using the static orifices and transducers described in the previous section. An electronic scanning pressure system was used to acquire the steady pressures, which were then converted to pressure coefficients. The uncertainty in $C_{P}$ is estimated to be \pm 0.02 . For the unsteady pressures, signals from the piezoelectric resistive transducers were amplified and then sent to the A/D converter where they were AC-coupled, low-pass filtered at $10 \mathrm{kHz}$, and digitized using a sample rate of $25.6 \mathrm{kHz}$ and sample duration of 32 seconds. To check the calibration coefficients and monitor the health of the transducers during the test, the signals for the transducers in the circumferential arrays were split before the A/D converter so the total signal could be digitized at a slower rate, averaged, and compared to the static pressure data. In general, this check showed that the transducers were quite stable and experienced little drift during the course of the test.

To help interpret the unsteady pressure data, flow visualization was performed to identify surface flow features. These features were visualized by applying a mixture of titanium dioxide $\left(\mathrm{TiO}_{2}\right)$ and kerosene to the model surface. As the tunnel was started and adjusted to the proper operating conditions, the mixture was transported along surface streaklines. Over time, the kerosene evaporated and the $\mathrm{TiO}_{2}$ was left on the surface in a solidified form, revealing the surface flow pattern.

\section{Discussion of Results}

\section{A. Consistency Between Phases}

One major concern in conducting multi-phase wind tunnel experiments is insuring that tunnel conditions and the physical features of the flow remain consistent between tunnel entries. Consistency is very important in this case since we desire to combine off-body information obtained via PIV from Phase 1 and surface flow information from Phase 2 to develop a comprehensive picture of the interference between tandem cylinders. There are some notable differences between the two phases of this study. Firstly, the cylinder diameter was increased in Phase 2 to accommodate the unsteady pressure transducers and secondly, the tunnel velocity was decreased to maintain the same Reynold's number. To assess consistency, $C_{P}$ distributions acquired on a single cylinder during each phase were compared. Figure 3, which was used to show the effectiveness of the transition strip, also shows the good agreement between the pressures acquired during the two phases. The only notable differences occur near the edges of the transition strips whose location is indicated by the two, gray vertical columns. To identify global differences in the flow between the two phases, the drag coefficient was computed by integrating the pressures measured on each cylinder between the limits $\theta=0 \mathrm{deg}$. and $\theta=360 \mathrm{deg}$. Figure 4 shows a comparison of the drag coefficients for each $\mathrm{L} / \mathrm{D}$ tested. The overall trends are consistent; however, the drag on the front cylinder is slightly higher in Phase 2. This is also the case for the rear cylinder but the differences occur mainly at L/D's between 3 and 4 . Despite these differences, the consistency was deemed adequate to characterize the flow at $\mathrm{L} / \mathrm{D}=1.435$ and $\mathrm{L} / \mathrm{D}=$ 3.7 using data from both phases.

\section{B. Flow Characterization}

Although several L/D spacings were tested, this discussion will focus on characterizing the flow associated with $\mathrm{L} / \mathrm{D}=1.435$ and $\mathrm{L} / \mathrm{D}=3.7$. For comparison, results for a single cylinder will also be presented. The flow field description will refer to the gap region and the near-wake region. The gap region includes flow on the rearward face of the front cylinder, on the forward face of the rear cylinder, and between the two cylinders. The near-wake region includes flow on the rearward face of the rear cylinder and the immediate vicinity downstream of the rear cylinder. For the PIV data presented, distances have been normalized by the cylinder diameter and are referenced to the center of the front cylinder for the gap flow figures and to the center of the rear cylinder for figures featuring the near-wake flow. Color contours of the spanwise vorticity have been normalized by the freestream velocity and cylinder diameter.

\section{Gap Region - Mean Flow}

The flow in the gap region is of primary importance because its interaction with the rear cylinder greatly influences the drag and the structure of the near-wake flow. Figures 5 and 6 show the mean flow structure in the gap region for $\mathrm{L} / \mathrm{D}=1.435$ and 3.7 using streamlines computed from the streamwise and vertical velocity components. In the figures, the streamlines have been superimposed over color contours of the streamwise velocity. For $\mathrm{L} / \mathrm{D}=$ 1.435 , the flow separates from the front cylinder and impinges on the rear cylinder to form a single cell of recirculating flow in the lower portion of the gap. Computations by Khorrami, et al. ${ }^{13}$ have shown that this type of 
flow pattern can result from flow angularity as small as 0.5 degrees. An extensive effort was made during both phases to align the model to the flow using both single and tandem cylinder configurations. The process was interative and involved adjusting the sideslip angle of the model in $0.1 \mathrm{deg}$. increments while checking the symmetry between pressures on the upper and lower surfaces of the cylinder. Even after finding the position that produced good symmetry for the single cylinder and $\mathrm{L} / \mathrm{D}=3.7$, the asymmetric flow pattern still persisted at $\mathrm{L} / \mathrm{D}=1.435$. The existence of this flow pattern in both phases of this study as well as in other experiments at both subcritical ${ }^{6}$ and supercritical $^{7}$ Reynolds numbers suggests that either there may be other contributing factors to the asymmetry besides sideslip angle or this may be a preferred flow state. In contrast, the mean flow pattern for $\mathrm{L} / \mathrm{D}=3.7$ is typical for a cylinder in a crossflow; namely, a region of recirculating flow behind the cylinder comprised of two, counter-rotating cells. For these particular cylinder spacings, the differences in the gap flow have a greater effect on the $C_{P}$ distribution for the rear cylinder than for the front cylinder. This is evident in Figs. 7 and 8 which show mean $C_{P}$ distributions for the front and rear cylinders. Despite slight differences in the pressure recovery region of the front cylinder, the $C_{P}$ distributions for $\mathrm{L} / \mathrm{D}=1.435$ and $\mathrm{L} / \mathrm{D}=3.7$ are almost identical. They both show a higher base pressure than the single cylinder and both show separation at $\theta=110 \mathrm{deg}$. and $\theta=250 \mathrm{deg}$. The $C_{P}$ distributions shown in Fig. 8 for the rear cylinder have radically different trends on the forward face between $\theta=0$ deg. and $\theta=90 \mathrm{deg}$. and $\theta=270 \mathrm{deg}$. and $\theta=360 \mathrm{deg}$. For $\mathrm{L} / \mathrm{D}=1.435$, the flow is dominated by the attachment of the shear layer and the associated flow bifurcation. For $\mathrm{L} / \mathrm{D}=3.7$, the flow stagnates at $\theta=0 \mathrm{deg}$. so the trend is similar to the front cylinder although the pressure level is lower. Differences are also noted on the rearward face of the cylinder between $\theta=130 \mathrm{deg}$. and $\theta=230 \mathrm{deg}$. Here, the pressure recovery is greater for $\mathrm{L} / \mathrm{D}=1.435$ than $\mathrm{L} / \mathrm{D}$ $=3.7$, which produces the negative drag coefficient shown in Fig. 4 .

In addition to streamlines and surface pressures, surface flow patterns shown in Figs. 9 and 10 provide further insight into the interaction of the gap flow with the cylinders. The photographs were taken with the camera perpendicular to the $\mathrm{X}-\mathrm{Z}$ plane and pointing in the negative $\mathrm{Y}$ direction. As such, the orientation of the cylinders differs from that shown in Figs. 5 and 6. The front cylinder is on the right, the rear cylinder is on the left, and the flow is from right to left. For $L / D=1.435$, the surface flow pattern on the front cylinder in Fig. 9 shows the transition strip (1) and streaks from the streamwise vortices (2) it generates. These vortices coalesce along the primary separation line (3). It also shows flow moving in the spanwise direction (4) between the primary separation line (3) and secondary separation line (5). Since the shear layers bound the flow, spanwise flow is necessary to maintain the momentum balance and may explain the streamline pattern near the upper shear layer in Fig. 5 where the recirculating flow traveling toward the front cylinder appears to change direction abruptly near $\mathrm{X} / \mathrm{D}=0.7$ and $\mathrm{Y} / \mathrm{D}=0.5$. For $\mathrm{L} / \mathrm{D}=3.7$, a similar surface flow pattern is seen on the front cylinder in Fig. 10 however there is little evidence of spanwise flow between the primary separation line (3) and secondary separation (5) line. If spanwise flow is characteristic of tandem configurations with small spacings, then computer simulations of such flows will have to be 3-D and have some estimate of the spanwise correlation length to predict these flows accurately.

For the rear cylinder, the surface flow patterns in Figs. 9 and 10 show little difference. The primary separation line (6) appears to occur at the same location for both configurations but shows more uniformity along the span of the cylinder for $\mathrm{L} / \mathrm{D}=3.7$. From the $C_{P}$ distributions shown in Fig. 8, the flow appears to separate near $\theta=120 \mathrm{deg}$. and $\theta=240 \mathrm{deg}$ for $\mathrm{L} / \mathrm{D}=1.435$ and near $\theta=130 \mathrm{deg}$. and $\theta=230 \mathrm{deg}$ for $\mathrm{L} / \mathrm{D}=3.7$. This is aft of the separation location estimated for the front cylinder. The difference in the separation location between the front and rear cylinders may be due to the differences in the turbulence levels in the boundary layer. The front cylinder, boundary layer undergoes transition but the rear cylinder, immersed in an unsteady flowfield, has a boundary layer that is already turbulent due to turbulent flow attaching to the forward surface.

\section{Gap Region - Unsteady Flow}

Full characterization of the unsteady flow in the gap region requires information about the instantaneous flow structure and how this structure interacts with the surface of the cylinders. Figures 11 and 12 compare the fluctuating pressure coefficients on a single cylinder to the two tandem configurations. The fluctuating pressure coefficient was computed by integrating the spectra across the frequency range to obtain the mean square value, taking the square root, and then normalizing by the freestream dynamic pressure. The relative uncertainty in the computed spectra and fluctuating pressure coefficient are estimated to be $10 \%$ and $5.4 \%$, respectively. Similar to the mean $\mathrm{Cp}$ distributions, Fig. 11 shows some variation between the three cases but in general, the unsteady gap flow appears to 
have minimal impact on the pressure fluctuations on the front cylinder at these conditions. The only effects are seen in the vicinity of $\theta=90^{\circ}$ and $\theta=270^{\circ}$ due to unsteadiness in the flow separation point and in the vicinity of $\theta=$ $135^{\circ}$ and $\theta=225^{\circ}$ where vortices formed behind the cylinder interact with the surface. Sun, et al. ${ }^{8}$ performed similar measurements on tandem cylinders at a supercritical Reynolds number of $6.5 \times 10^{5}$ and found that the fluctuating pressures on the front cylinder collapsed into a single curve for $\mathrm{L} / \mathrm{D} \leq 4$. Although the curves in this study did not collapse, they do show similar trends and only slight variations in pressure level. In addition to the pressure distributions in Fig. 3, this may provide further evidence of the transition strip's effectiveness in producing post-critical flow on the cylinders.

Compared to the front cylinder, the effects of unsteady flow in the gap region on the rear cylinder are more pronounced. Overall, the fluctuating pressure levels in Fig. 12 are higher for $\mathrm{L} / \mathrm{D}=3.7$ than for $\mathrm{L} / \mathrm{D}=1.435$. For the former case, the maximum value of $C_{P}{ }^{\prime}$ measured using the circumferential tranducers is 0.68 and occurs in the vicinity of $\theta=45^{\circ}$ and $\theta=315^{\circ}$. The maximum levels for $\mathrm{L} / \mathrm{D}=1.435$ are 0.14 and 0.18 and occur in the vicinity of $\theta=60^{\circ}$ and $\theta=300^{\circ}$ where the shear layers attach to the rear cylinder. These surface flow characteristics are corroborated by representative frames of the instantaneous spanwise vorticity shown in Figs. 13 and 14. Figure 13 shows the attachment of the lower shear layer on the rear cylinder for $\mathrm{L} / \mathrm{D}=1.435$ and Fig. 14 shows the formation of a vortex and the interaction of the vortex sheet with the rear cylinder for $\mathrm{L} / \mathrm{D}=3.7$. In both cases, smaller vortical structures formed in the shear layer can be seen in proximity of the rear cylinder, which could possibly contribute to higher surface pressure fluctuations and broader peaks in the spectra.

Spectra for individual transducers on the front and rear cylinders at $\mathrm{L} / \mathrm{D}=1.435$ and $\mathrm{L} / \mathrm{D}=3.7$ were examined to identify frequencies associated with features such as flow separation, shear layer attachment, and vortex shedding. Figures 15 and 16 show pressure power spectra computed for transducers around the circumference of the front and rear cylinders. Since the spectra are fairly symmetric between the upper and lower halves of each cylinder, spectra at $\theta=90,135$, and $180 \mathrm{deg}$. on the front cylinder and $\theta=0,45$, and $90 \mathrm{deg}$. on the rear cylinder have been compared in Figs. 17-20 for this discussion.

On the front cylinder, the spectra for $\mathrm{L} / \mathrm{D}=1.435$ in Fig. 17 and the spectra for $\mathrm{L} / \mathrm{D}=3.7$ in Fig. 18 have comparable energy levels and features with the exception of the peaks in Fig. 18 associated with vortex shedding. At $\theta=90$ deg., the pressure level increases between $1 \mathrm{kHz}$ and $10 \mathrm{kHz}$. After examining the surface flow patterns, this increase is believed to be due to the streamwise vortices generated by the transition strip as identified in Fig. 9. In comparison, the spectra on the rear cylinder in Figs. 19 and 20 at $\theta=90$ deg. do not show this feature. At $\theta=135$ deg., the spectra for both configurations show a broadband "hump" centered at $1 \mathrm{kHz}$. Although not shown in this paper, the spectra for the transducers in the spanwise array at this same azimuth all have this feature as well. It was also seen in spectra from a hot-wire survey conducted through the shear layer at $\mathrm{X} / \mathrm{D}=0.5$ during Phase 1 and appears in the spectrum on the rear cylinder at $\theta=45 \mathrm{deg}$., which is near the location where the flow attaches. This provides conclusive evidence that this hump is associated with the shear layer. Based on an examination of instantaneous PIV images, it is unclear whether the hump is reflecting the movement of the shear layer, the vortical structures of various scales being convected, or both. In addition to the "hump", Fig. 18 also shows peaks at 190.6 $\mathrm{Hz}$ and $381 \mathrm{~Hz}$ associated with vortex shedding from the front cylinder. For both configurations, the spectra at $\theta=$ $180 \mathrm{deg}$. are rather benign.

Spectra in Figs. 19 and 20 for the rear cylinder show similar features but the pressure levels are much higher than those measured on the front cylinder over the entire frequency range. For $\mathrm{L} / \mathrm{D}=1.435$, the "humps" are present at both $\theta=45^{\circ}$ and $\theta=90^{\circ}$ but the energy level is higher at $\theta=45^{\circ}$ due to its proximity to the attachment point. For $\mathrm{L} / \mathrm{D}=3.7$, peaks associated with vortex shedding as well as harmonics of this frequency show up in the each of the sensors. At $\theta=0^{\circ}$, more energy is in the harmonic peak than in the primary shedding peak. Since this transducer is located at the centerline between the front and rear cylinders, it senses the vortices shed from both sides of the front cylinder instead of just one. Similar features were seen in hot-wire spectra from traverses behind the rear cylinder. 


\section{Near-Wake Region}

Although the near-wake behind the rear cylinder is assumed to be dominated by vortex shedding, the spacing between the cylinders and consequently the gap flow affects the mean and unsteady wake structure as well. This is evident in Figs. 21a-c and Figs. 22a-c which show mean streamlines and a representative frame of the instantaneous spanwise vorticity behind a single cylinder and the rear cylinder of the two tandem configurations. In Fig. 21 the size of the recirculation bubble in terms of width and length differs between the three configurations due to differences in separation location discussed earlier. Additional flow field measurements at other L/D's are needed to determine if the wake width and closure length are constant for a given flow regime. The size of this region has implications on pressure recovery and indicates where the unsteady flow is most likely to interact with the cylinder surface.

In terms of the unsteady wake structure, Figs. 22a-c show that the wake is more organized for the single cylinder and $L / D=3.7$ than for $L / D=1.435$. From the mean streamlines and steady surface pressures, it is apparent that for $\mathrm{L} / \mathrm{D}=1.435$ flow acceleration around the rear cylinder is suppressed, and consequently, pressure recovery is reduced and base pressure is higher. This, in turn, may lead to a less organized vortex street in the wake, as both the positive and negative levels of vorticity are reduced. Lower vorticity levels would explain why vortex shedding peaks are absent from the spectra in Fig. 19 for $\mathrm{L} / \mathrm{D}=1.435$. To investigate this further, pressure spectra acquired on the rear cylinder at $\theta=225 \mathrm{deg}$. and velocity spectra acquired during Phase 1 downstream of the rear cylinder were plotted versus Strouhal number. Figure 23 shows the measurement locations relative to the rear cylinder and Figs. 24 and 25 show pressure and velocity spectra. Both measurement techniques capture the shedding from the single cylinder and $\mathrm{L} / \mathrm{D}=3.7$ but for $\mathrm{L} / \mathrm{D}=1.435$, no narrowband peaks appear in the pressure spectra. A broadband peak is distinguishable in the velocity spectra at $S t=0.280$ but it is unclear from the experimental data whether this is due to a less-organized vortex sheet or due to "undulations" in the shear layer. An examination of additional frames of instantaneous spanwise vorticity did not show evidence of vortex formation behind the rear cylinder for $\mathrm{L} / \mathrm{D}=1.435$ but did show the type of undulations seen in free shear layers. These results indicate that the interference effects for the $\mathrm{L} / \mathrm{D}=1.435$ configuration, and perhaps other configurations with small spacings, can be considerable and the assumption that it sheds like a single cylinder may be invalid.

In addition to spectra, the pressure correlation length is an important parameter for characterizing unsteady flow on cylinders. Not only does it indicate the distance over which the surface pressure fluctuations are correlated, but it also provides information about vortex shedding. In research on single cylinders, Bruun, et al. ${ }^{20}$ found that longer correlation lengths (i.e., distances over which correlation coefficients are significant) are indicative of coherent vortex shedding continuing over longer distances downstream from the generating cylinder. Conversely, shorter correlation lengths indicate smaller distances over which the wake vortices stay coherent.

The broadband correlation length was computed on the single cylinder and rear cylinder of the tandem configurations using the spanwise row of transducers at $\theta=135^{\circ}$. Starting with the transducer closest to the floor of the tunnel as the reference point, the correlation coefficient was computed between it and each transducer above it. This process was repeated using each successive transducer as the reference point until correlations had been computed for every possible transducer combination. In Figs. 26 and 27, the correlation coefficients computed using different reference points along the span of the cylinder have been plotted for $\mathrm{L} / \mathrm{D}=1.435$ and $\mathrm{L} / \mathrm{D}=3.7$. The correlation length for $\mathrm{L} / \mathrm{D}=1.435$ is much shorter than for $\mathrm{L} / \mathrm{D}=3.7$. This is indicative of a wake that shows coherent vortex shedding over a very short distance. The correlation length for $\mathrm{L} / \mathrm{D}=3.7$, on the other hand, is much longer, indicating coherent vortex shedding over longer distances downstream. This is confirmed by the PIV images shown in Fig. 22. The two plots also provide insight into the dependence of the correlation, and consequently correlation length, on the location of the reference transducer. For $\mathrm{L} / \mathrm{D}=1.435$, the curves collapse indicating that the correlation is independent of the location of the reference point. In contrast, the curves for $\mathrm{L} / \mathrm{D}=3.7$ begin to diverge after $\mathrm{Z} / \mathrm{D}=1.25$ which shows the dependence of correlation on the location of the reference point and suggests that the flow may be nonuniform along the cylinder span. Howell and Novak ${ }^{21}$ documented similar results for a single cylinder but also found that the correlation length was dependent on end effects. Although further analysis is needed, this may explain the differences in Fig. 27 given that the points where the curves differ most correspond with transducers located near the tunnel ceiling. 
To examine the relationship between correlation lengths based on pressure fluctuations and correlation lengths based on velocity fluctuations, results from the current phase are compared to correlations from Phase 1 in Fig. 28. The fluctuating velocities were measured using two hot wires at the same location behind the rear cylinder where the velocity spectra were acquired. The first hot wire was fixed at mid-span while the second hot-wire was traversed toward the tunnel ceiling. For the fluctuating pressure correlations, the transducer at mid-span was used as the reference point. With the exception of $\mathrm{L} / \mathrm{D}=1.435$, the velocity correlations in Fig. 28 are lower than the pressure correlations, at a given separation, indicating a shorter correlation length. This is to be expected due to vortex shedding and increased three-dimensionality of the flow as the wake develops. The differences between the correlations for $\mathrm{L} / \mathrm{D}=1.435$ may be due to spanwise flow on the surface of the rear cylinder between the primary and secondary separation lines.

\section{Conclusion}

A multi-phase experimental study has been conducted to characterize the unsteady interaction between two cylinders in tandem arrangement. Transition strips applied to the upper and lower surface of the front cylinder were successful in achieving post-critical flow around the cylinder. Steady and unsteady surface pressures, flow visualization, PIV, and hot-wire anemometry have been used to establish a comprehensive dataset for the flow around a single cylinder and a tandem configuration for $\mathrm{L} / \mathrm{D}=1.435$ and $\mathrm{L} / \mathrm{D}=3.70$. Characteristics about the flow in the gap and near-wake regions are summarized below.

1. Mean flow in the gap region at $\mathrm{L} / \mathrm{D}=1.435$ exhibited a single recirculation cell and impingement of the free shear layer on the front of the rear cylinder. Significant spanwise flow appeared to occur between the primary and secondary separation locations. At $\mathrm{L} / \mathrm{D}=3.7$, two counter-rotating recirculation cells were generated, and stagnation occurred on the front face of the rear cylinder at $\theta=0 \mathrm{deg}$. There was little evidence of spanwise flow between the primary and secondary separation locations. Separation on the rear cylinder appeared to occur about 10 degrees further forward for $\mathrm{L} / \mathrm{D}=1.435$ than for $\mathrm{L} / \mathrm{D}=3.7$.

2. Unsteady flow in the gap region was characterized by much higher unsteady pressures on the forward face of the rear cylinder than on the rearward face of the front cylinder, due, most likely, to the impingement of a turbulent shear layer on the rear cylinder. Power spectra from transducers on the surface of the front cylinder showed peaks at vortex shedding frequencies for the single cylinder and $\mathrm{L} / \mathrm{D}=3.7$. Vortex shedding for $\mathrm{L} / \mathrm{D}=1.435$ was suppressed. There was also evidence of increased spectra levels at high frequencies, at $\theta=90$ and $\theta=270 \mathrm{deg}$, possibly due to the streamwise vorticies shed by the transition strips, as seen in the surface flow visualization. Spectra levels on the forward face of the rear cylinder were 3 to 5 orders of magnitude greater than on the front cylinder, likely due to the impingement of the turbulent shear layer separated from the front cylinder.

3. Flow in the near-wake region was characterized by vortex shedding. However, the wakes for the single cylinder and rear cylinder at $L / D=3.7$ were more organized than for $L / D=1.435$. Acceleration around the rear cylinder for this L/D was suppressed, pressure recovery was reduced, and the base pressure was higher, which may have contributed to the lack of organization in the wake. No evidence of a vortex shedding peak in the spectra for this L/D existed. The existence of a slight "hump" in the spectra measured by a hot wire in the wake is surmised to be due to an "undulation" of the shear layers rather than a real vortical structure. Correlation lengths on the rear cylinder for $\mathrm{L} / \mathrm{D}=3.7$ are approximately equal to the single cylinder whereas the correlation length for $\mathrm{L} / \mathrm{D}=1.435$ is shorter. Of the two tandem configurations discussed, the near-wake structure for $\mathrm{L} / \mathrm{D}=3.7$ is most like that of a single cylinder.

These results, along with those obtained during the other phases of this study, will be used to establish a comprehensive dataset consisting of surface and off-body measurements on a tandem cylinder configuration. Such data can be used to 1) characterize the interaction of the wake of the front cylinder with the rear cylinder for different spacings, 2) examine differences between the wake of the entire tandem configuration and a single cylinder due to this interaction and 3) develop and validate computational techniques for the prediction of unsteady flows. 


\section{Acknowledgments}

The authors express their appreciation to Mr. Richard White, Mr. Al Barnes, and Mr. Marion Martin for their assistance with the experimental setup, instrumentation, and data acquisition; Mr. Jerome Harris and Dr. ChungSheng Yao for assisting with the PIV measurements; Mr. Tom Hall and Mr. Mick Hartzheim for installation and wiring of the pressure transducers; and to Mr. Floyd Backley and Mr. Tim Wood for the model design and fabrication.

\section{References}

${ }^{1}$ Lee, T. and Basu, S., "Nonintrusive Measurements of the Boundary Layer Developing on a Single and Two Circular Cylinders," Experiments in Fluids, 23, 1997, pp. 187-192.

${ }^{2}$ Itoh, Y. and Himeno, R., "Numerical Simulation of Three-Dimensional Flow Around Two Circular Cylinders in Tandem Arrangement," Focused on High Performance Computing in RIKEN 2001, RIKEN Review No. 48, October, 2002.

${ }^{3}$ Alam, M. M., Moriya, M., Takai, K., and Sakamoto, H., "Fluctuation Fluid Forces Acting on Two Circular Cylinders in a Tandem Arrangement at a Subcritical Reynolds Number," Journal of Wind Engineering and Industrial Aerodynamics, 91, 2003, pp. 139-154.

${ }^{4}$ Zdravkovich, M. M., "Review of Flow Interference Between Two Circular Cylinders in Various Arrangements," Journal of Fluids Engineering, Vol. 99, Dec. 1977, pp 618-633.

${ }^{5}$ Zdravkovich, M.M., "Flow Induced Oscillations of Two Interfering Circular Cylinders," J. of Sound and Vibrations, Vol. 101, No.4, pp. 511-521, 1985.

${ }^{6}$ Lin, J.-C., Yang, Y. And Rockwell, D., Flow Past Two Cylinders in Tandem: Instantaneous and Average Flow Structure," Journal f Fluids and Structures, Vol. 16, Issue 8, 2002, pp. 1059-1071.

${ }^{7} \mathrm{Gu}, \mathrm{Z}$., "On Interference Between Two Circular Cylinders at Supercritical Reynolds Number," Journal of Wind Engineering and Industrial Aerodynamics, 62, 1996, pp. 175-190.

${ }^{8}$ Sun, T.F., Gu, Z.F., He, D.X., and Zhang, L.L., "Fluctuating Pressure on Two Circular Cylinders at High Reynolds Numbers," Journal of Wind Engineering and Industrial Aerodynamics, 41-44, 1992, pp. 577-588.

${ }^{9}$ Gu, Z.F., Sun, T.F., He, D.X., and Zhang, L.L., "Two Circular Cylinders in High-Turbulence Flow at Supercritical Reynolds number," Journal of Wind Engineering and Industrial Aerodynamics, 49, 1993, pp. 379-388.

${ }^{10}$ Okajima, A., "Flow Around Two Tandem Circular Cylinders at Very High Reynolds Numbers," Bulletin of the JSME, Vol. 22, No. 166, April 1976, pp. 504-511.

${ }^{11}$ Ljungkrona, L, Norberg, C., and Sunden, B., "Free-stream Turbulence and Tube Spacing Effects on Surface Pressure Fluctuations for Two Tubes in an In-line Arrangement," Journal of Fluids and Structures, Vol. 5, 1991, pp. $701-727$.

${ }^{12}$ Jenkins, L. N., Khorrami, M. R., Choudhari, M. M., and McGinley, C. B., "Characterization Of Unsteady Flow Structures Around Tandem Cylinders For Component Interaction Studies In Airframe Noise,”, AIAA-2005-2812, 2005.

${ }^{13}$ Khorrami, M. R., Choudhari, M. M., Jenkins, L. N. and McGinley, C. B., "Unsteady Flowfield Around Tandem Cylinders as Prototype for Component Interaction in Airframe Noise," AIAA-2005-2866, 2005.

${ }^{14}$ Hutcheson, F. V. and Brooks, T. F., "Noise radiation from Single and Multiple Rod Configurations," AIAA- 2006-2629, 2006.

${ }^{15}$ Sellers, W. L. and Kjelgaard, S. O., "The Basic Aerodynamic Research Tunnel - A Facility Dedicated to Code Validation," AIAA-88-1997, May 1988

${ }^{16}$ Washburn, A. E., "The Effect of Freestream Turbulence on the Vortical Flow over a Delta Wing," Master of Science Thesis, George Washington University, 1990.

${ }^{17}$ Jenkins, L. N., "An Experimental Investigation of the Flow Over a Notchback Automobile Configuration," Master of Science Thesis, George Washington University, 1999.

${ }^{18}$ Pierce, Allan D.: Acoustics: An Introduction to Its Physical Principles and Applications. Acoustical Society of America, Melville, NY. 1989.

${ }^{19}$ Roshko, A. "Experiments on the Flow Past a Circular Cylinder At Very High Reynolds Number," Journal of Fluid Mechanics, Vol. 10, 1961, pp 345-356.

${ }^{20}$ Bruun, H.H, and Davies, P.O.A.L., "An Experimental Investigation of the Unsteady Pressure Forces on a Circular Cylinder in a Turbulent Cross Flow," J. of Sound and Vibration, Vol. 40, No.4, pp. 535-559, 1975.

${ }^{21}$ Howell, J.F. and Novak, M., "Vortex Shedding From Circular Cylinders in Turbulent Flow," Wind Engineering Proceedings of the Fifth International Conference, edited by J.E. Cermak, Vol 1, July 1979, pp. 619-629, 
Figures

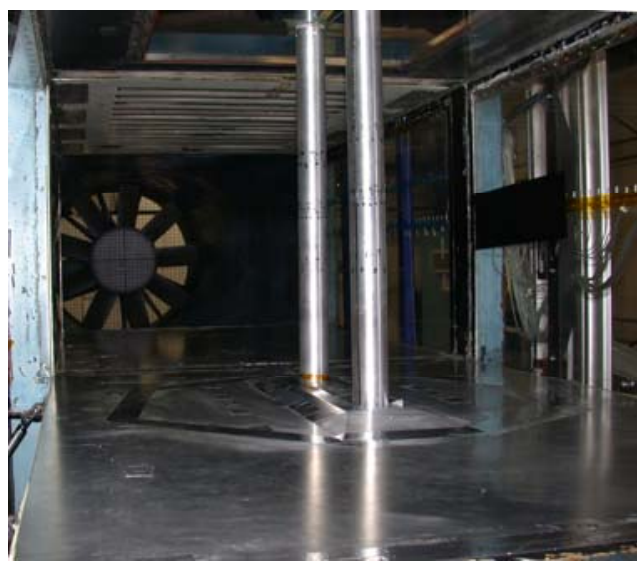

Figure 1. Tandem cylinder configuration in the BART facility.
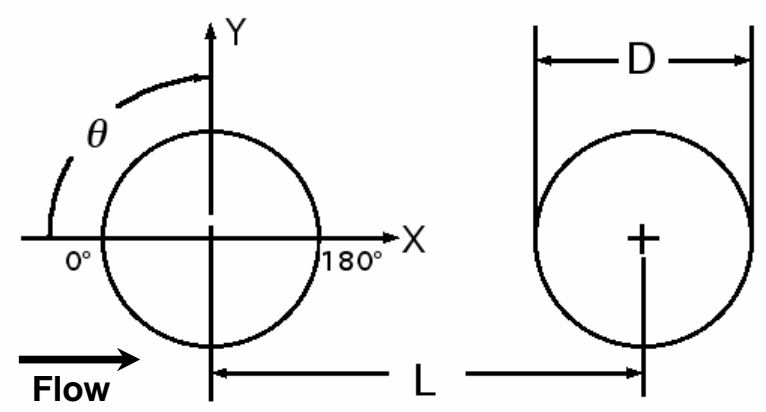

Figure 2. Model schematic and coordinate reference system.

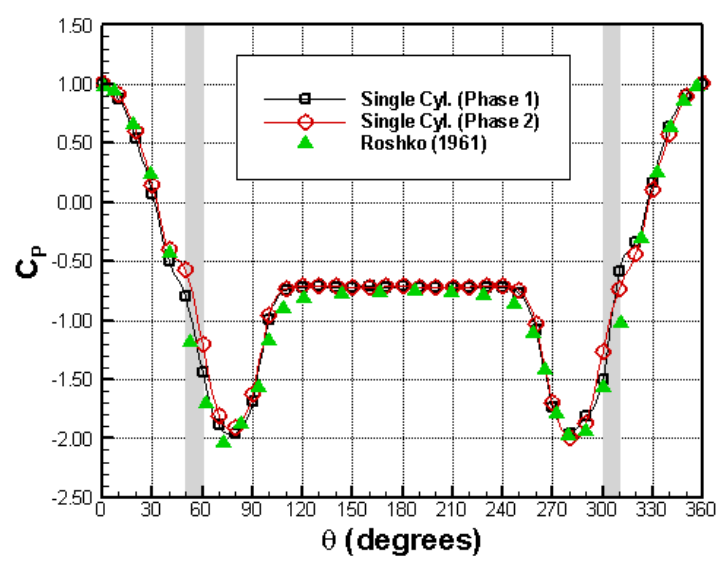

Figure 3. Pressure distribution on a single cylinder with transition strip.

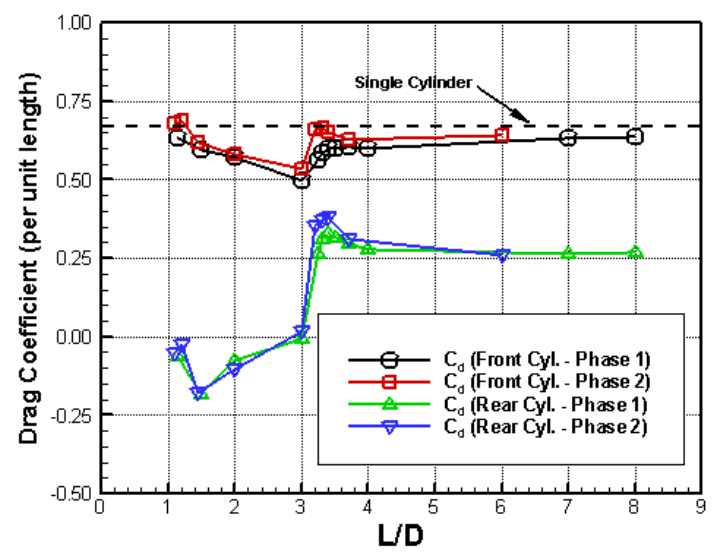

Figure 4. Effect of cylinder spacing on drag coefficient. 


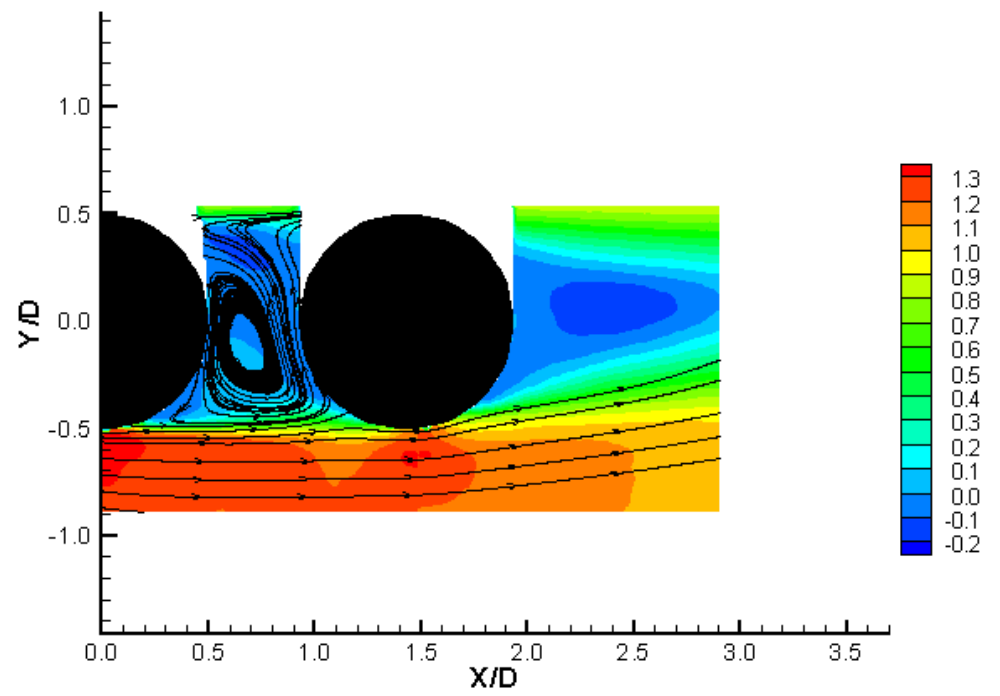

Figure 5. Mean streamlines in gap region $(L / D=1.435)$.

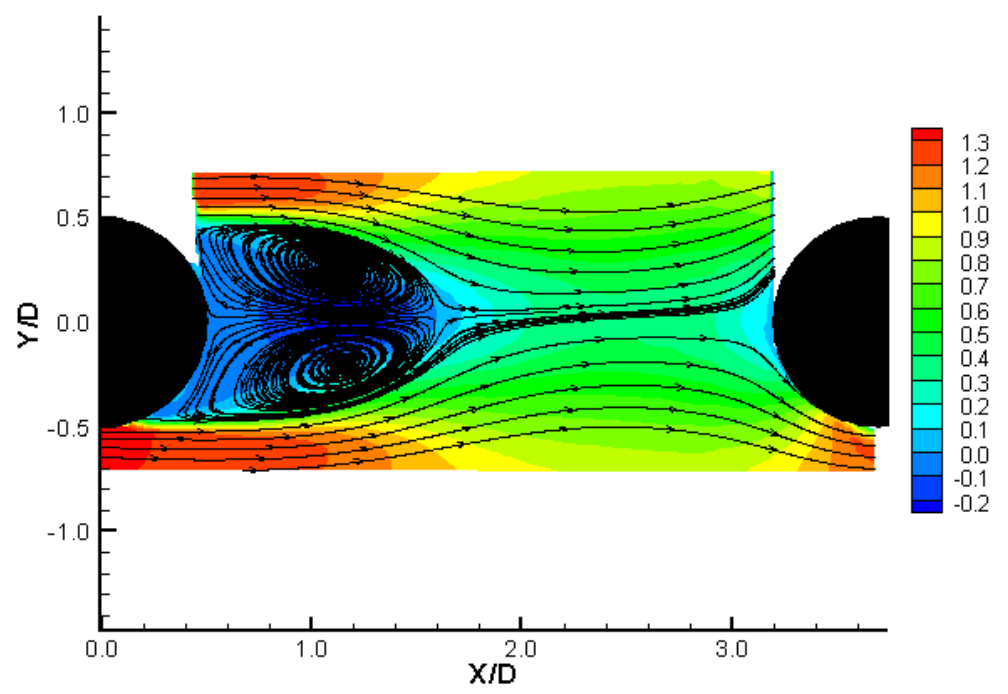

Figure 6. Mean streamlines in gap region $(\mathrm{L} / \mathrm{D}=3.7)$.

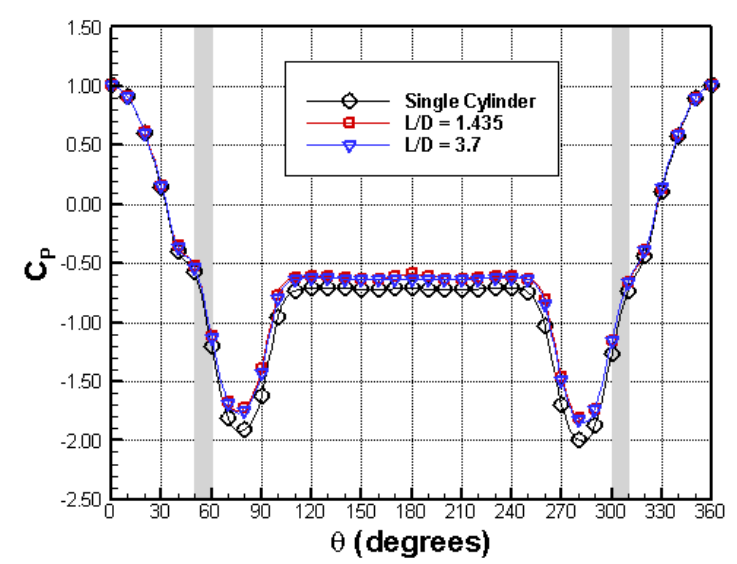

Figure 7. Mean pressures on front cylinder.

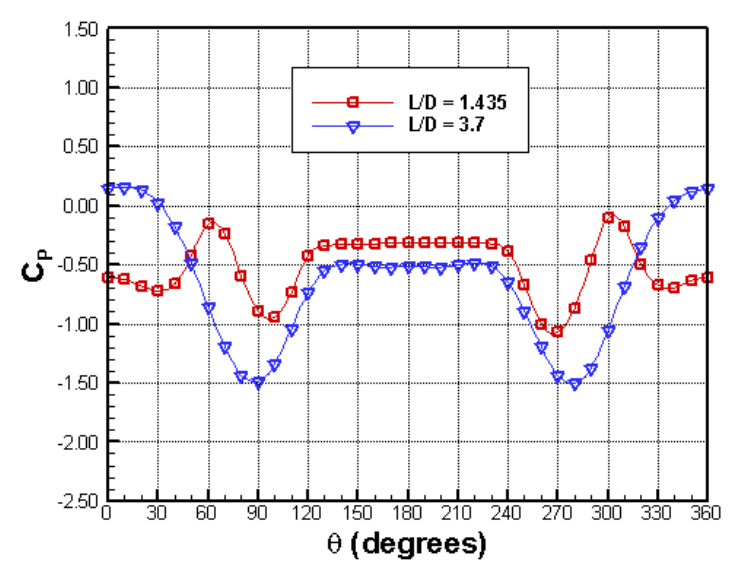

Figure 8. Mean pressures on rear cylinder. 


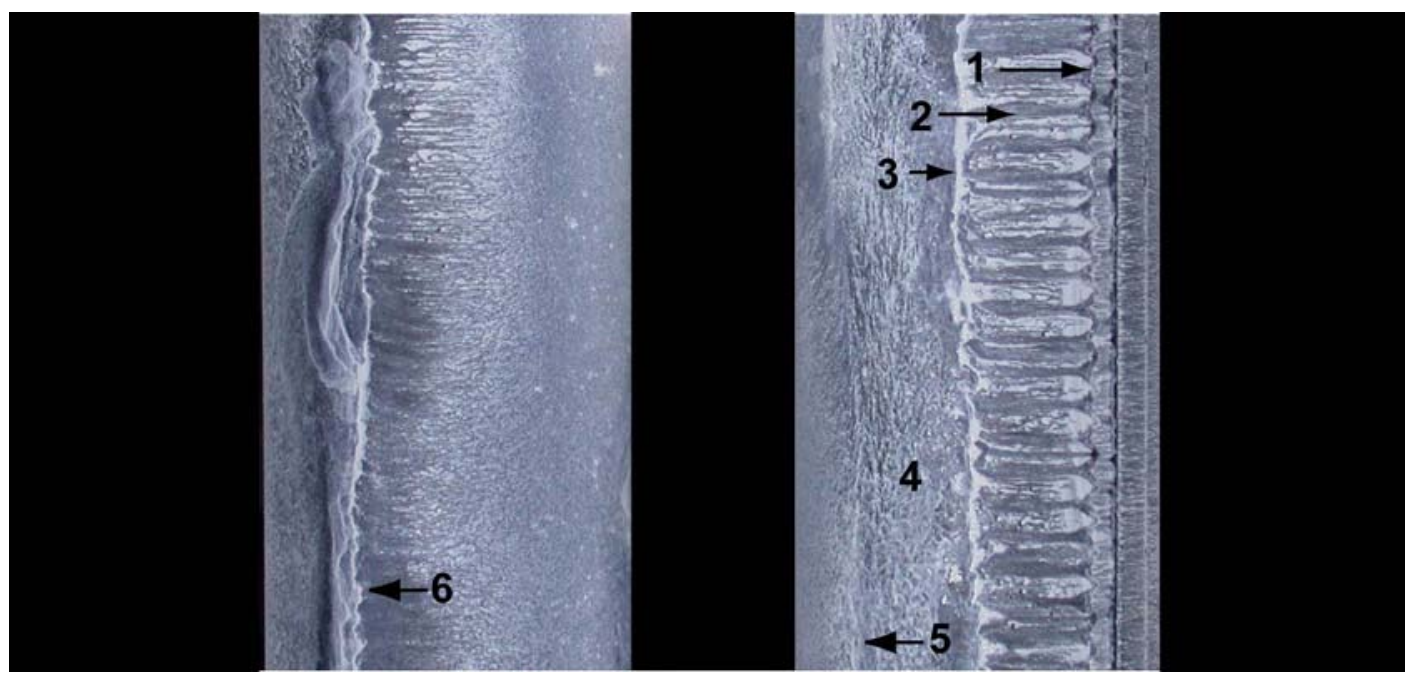

Figure 9. Surface flow visualization $(L / D=1.435)$.

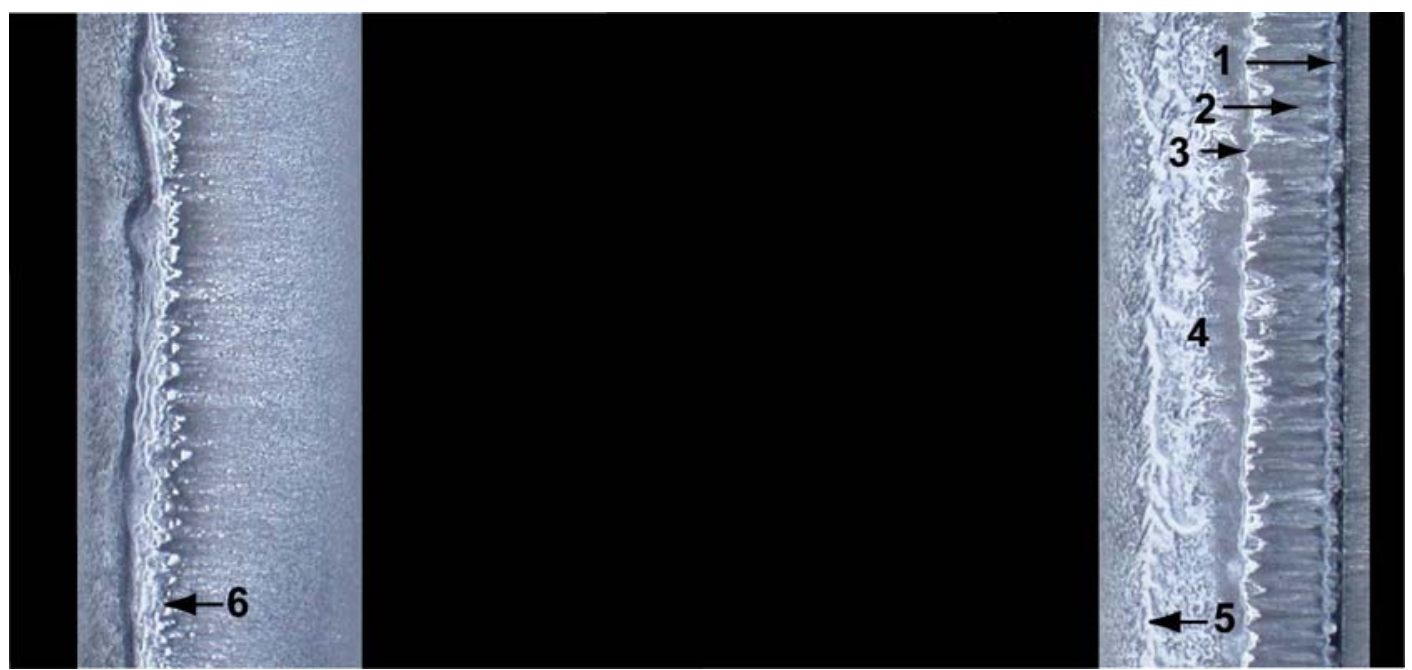

Figure 10. Surface flow visualization $(\mathrm{L} / \mathrm{D}=3.7)$.

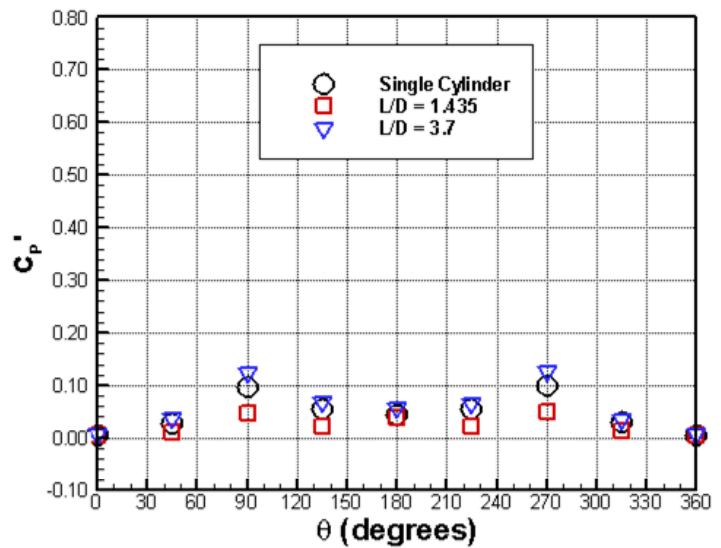

Figure 11. Fluctuating pressure coefficient on front cylinder.

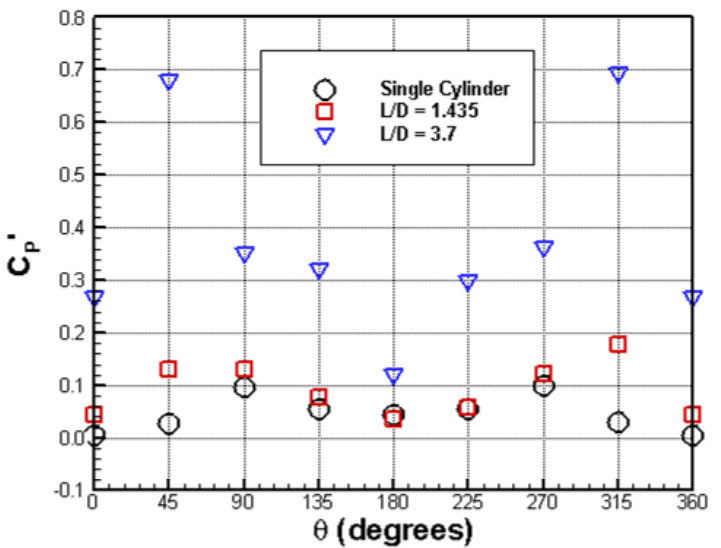

Figure 12. Fluctuating pressure coefficient on rear cylinder. 


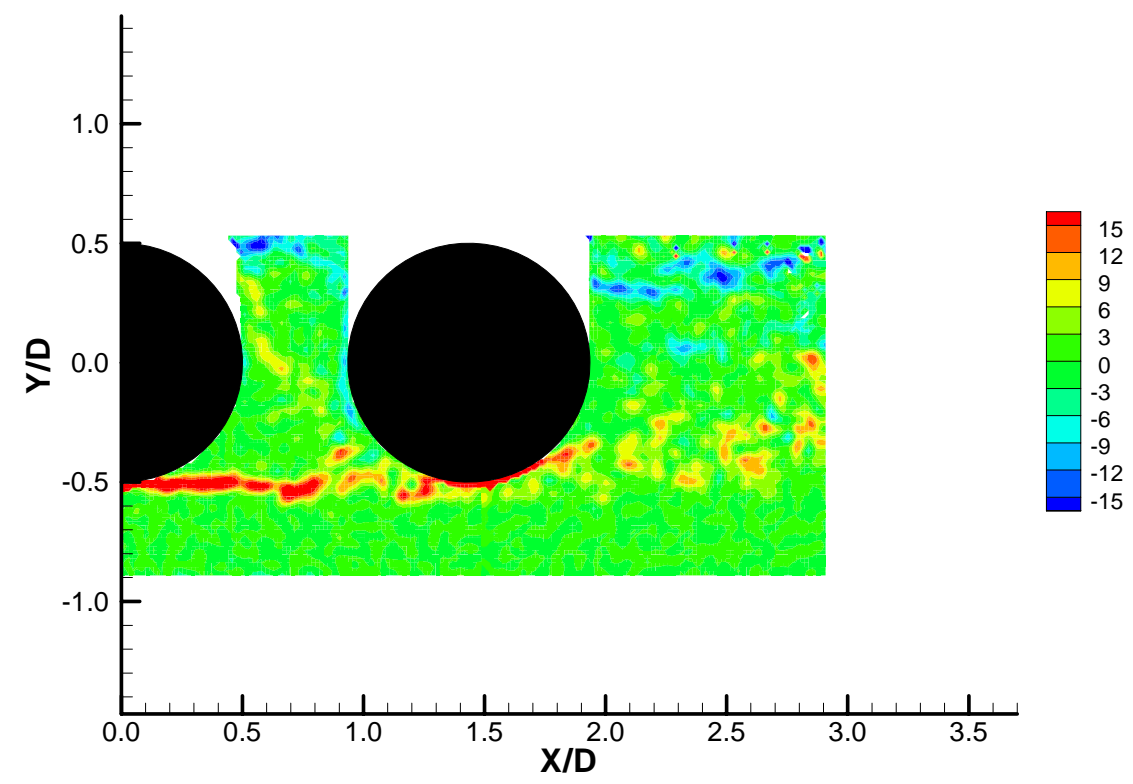

Figure 13. Instantaneous spanwise vorticity $(\mathrm{L} / \mathrm{D}=1.435)$.

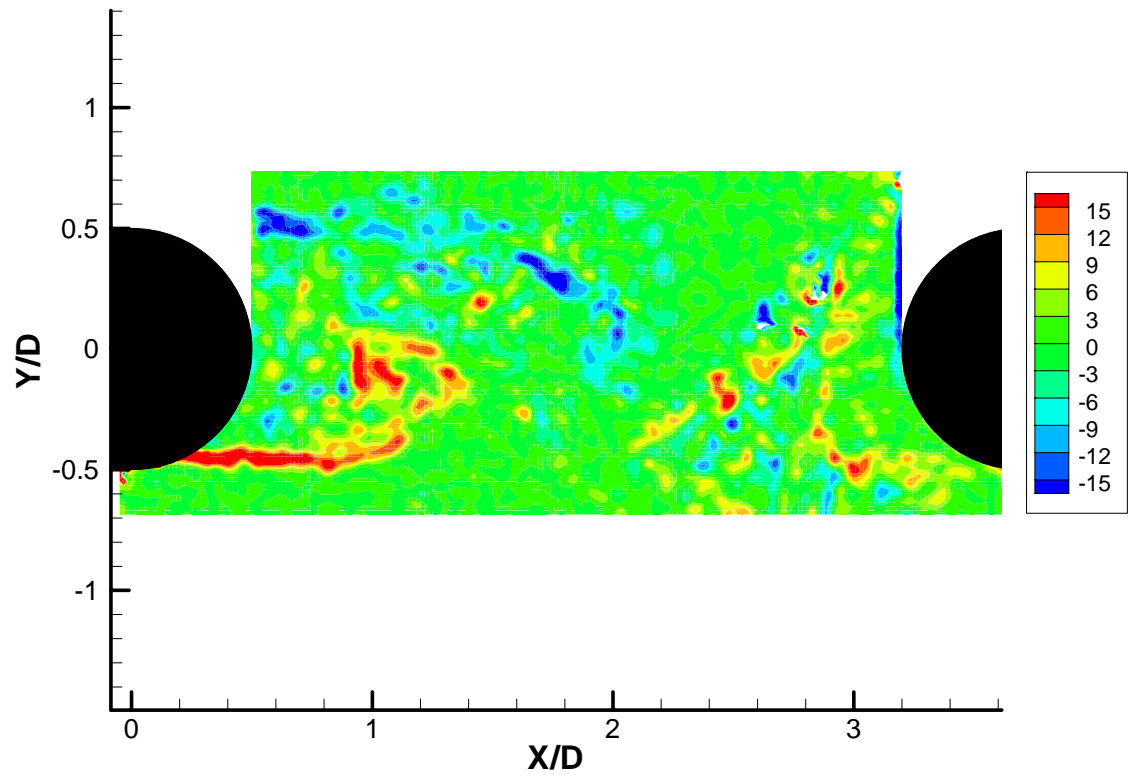

Figure 14. Instantaneous spanwise vorticity $(\mathrm{L} / \mathrm{D}=3.70)$. 


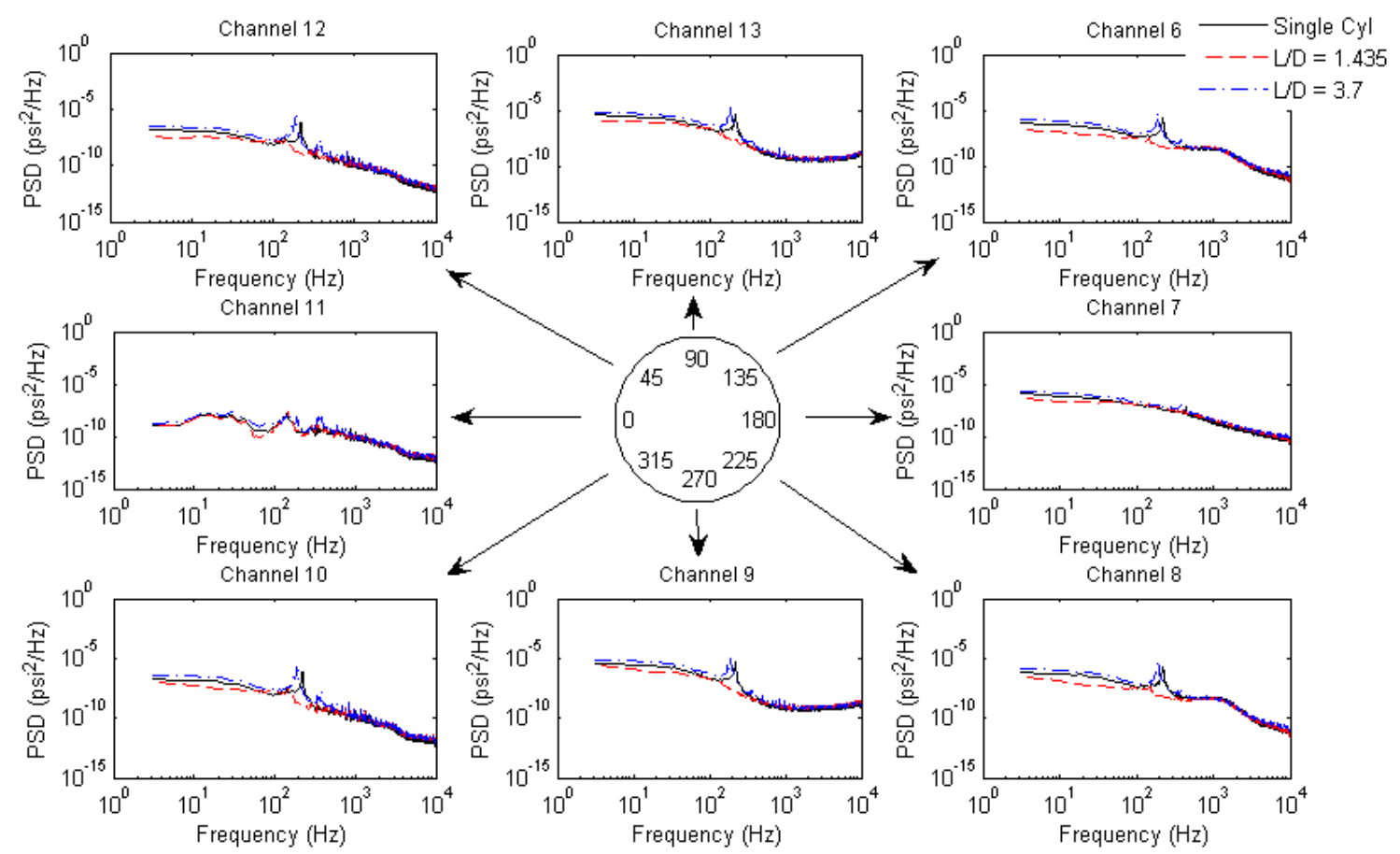

Figure 15. Pressure power spectra on front cylinder for $L / D=1.435$ and $L / D=3.7$ compared to single cylinder.

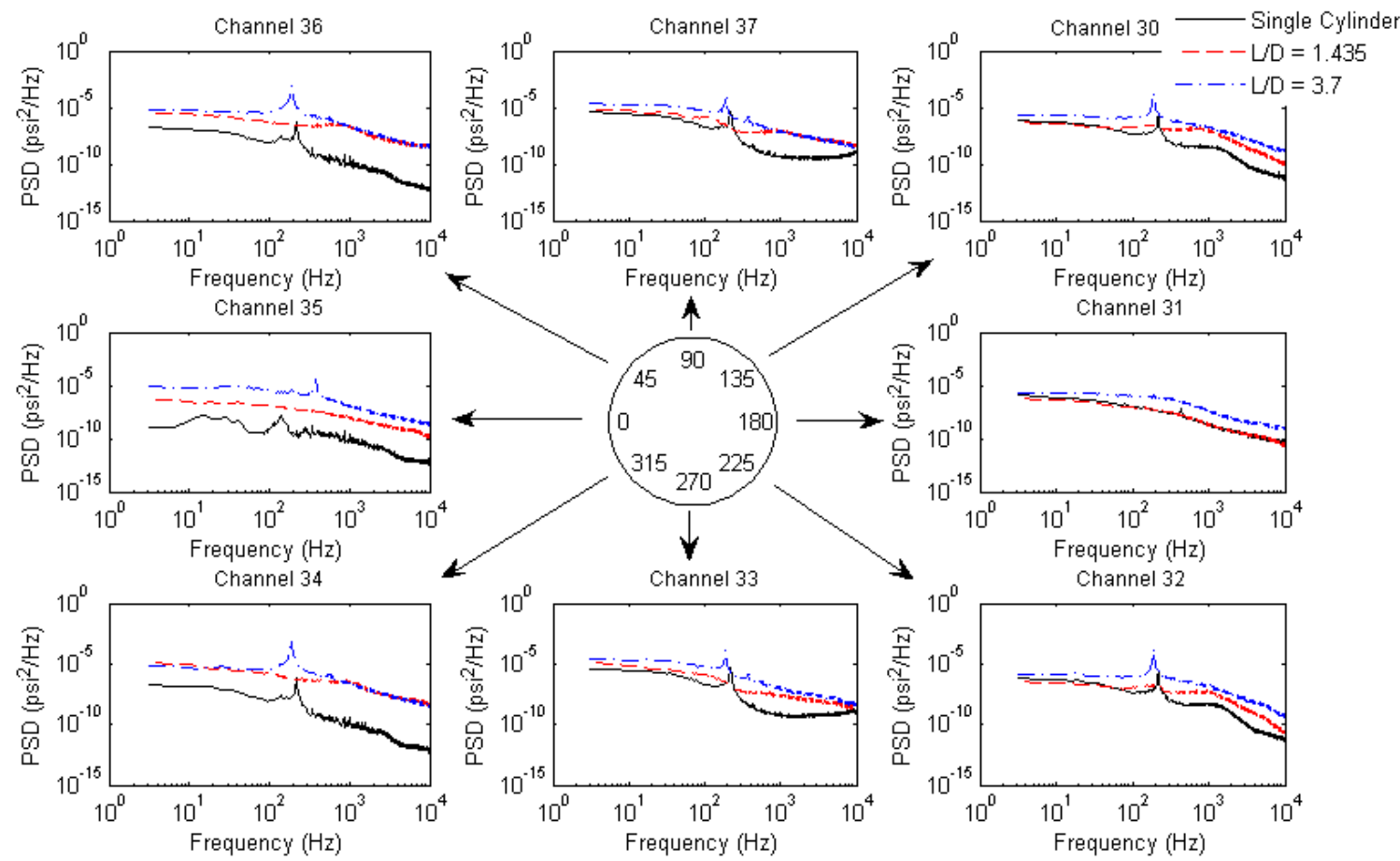

Figure 16. Pressure power spectra on rear cylinder for $L / D=1.435$ and $L / D=3.7$ compared to single cylinder. 


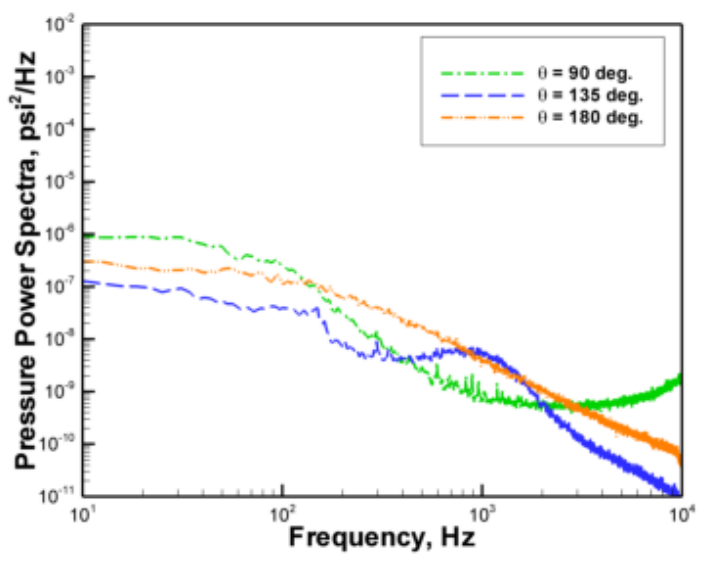

Figure 17. Spectra on front cylinder $(\mathrm{L} / \mathrm{D}=1.435)$.

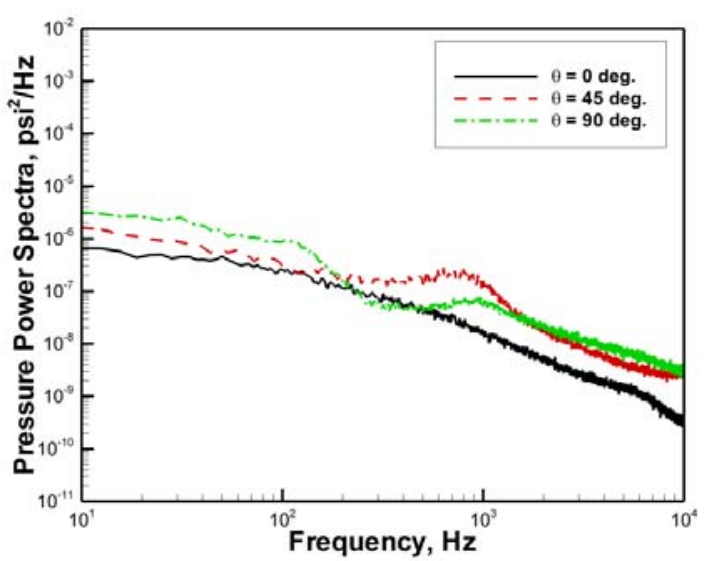

Figure 19. Spectra on rear cylinder $(L / D=1.435)$.

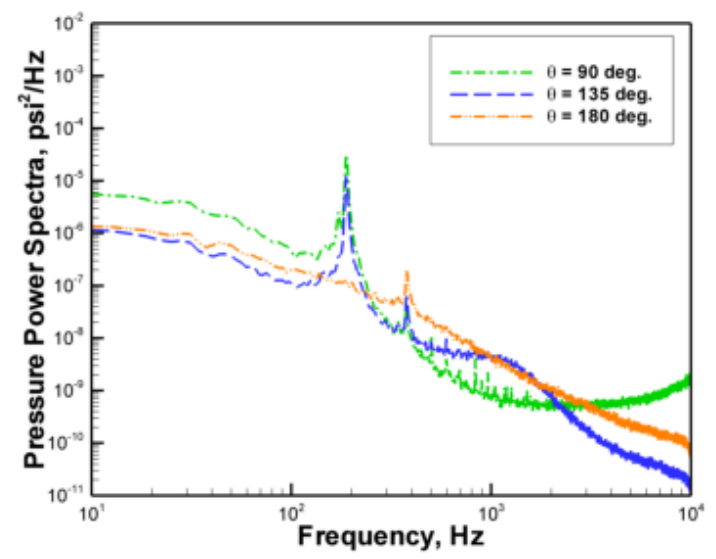

Figure 18. Spectra on front cylinder $(\mathrm{L} / \mathrm{D}=3.7)$.

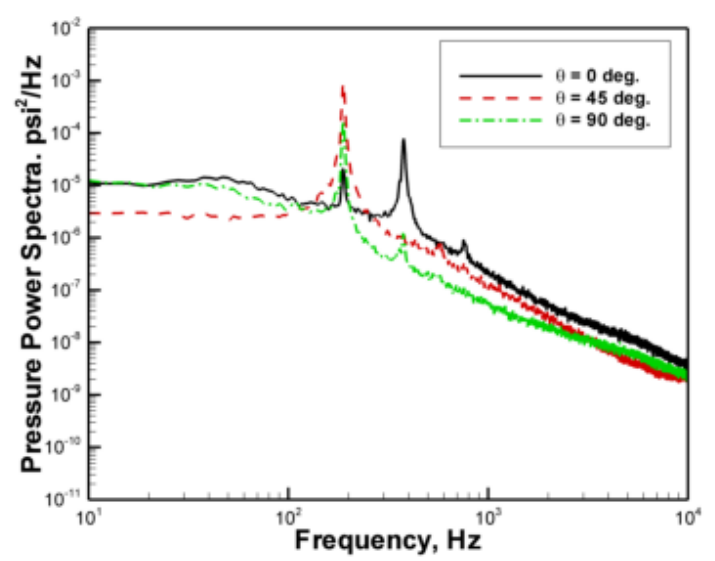

Figure 20. Spectra on rear cylinder $(\mathrm{L} / \mathrm{D}=3.7)$. 


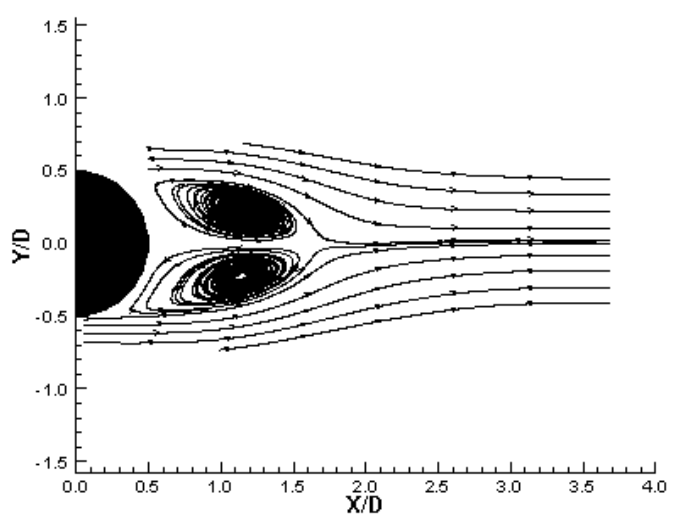

a) Single cylinder

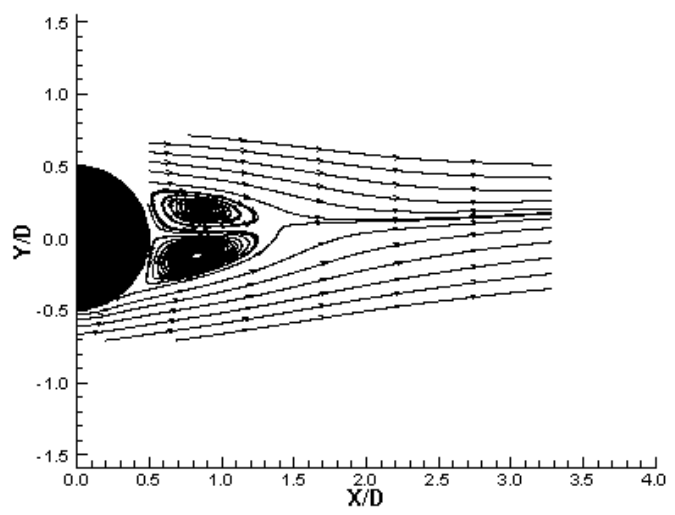

b) $\mathrm{L} / \mathrm{D}=1.435$

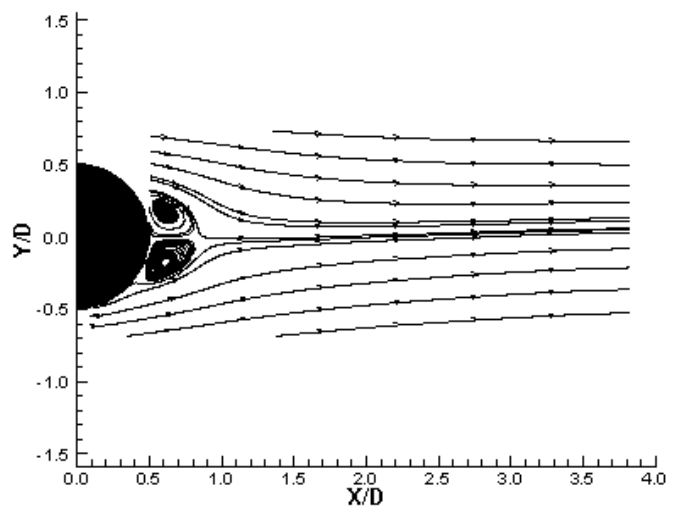

c) $\mathrm{L} / \mathrm{D}=3.70$

Figure 21. Mean streamlines in near-wake region

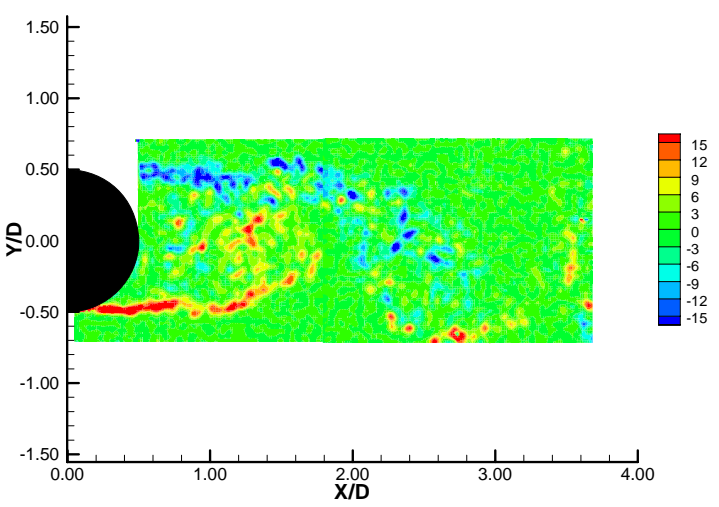

a) Single cylinder

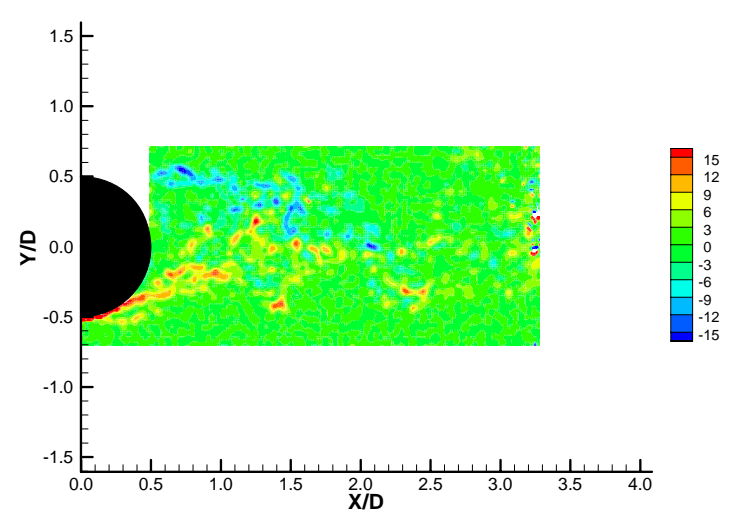

b) $\mathrm{L} / \mathrm{D}=1.435$

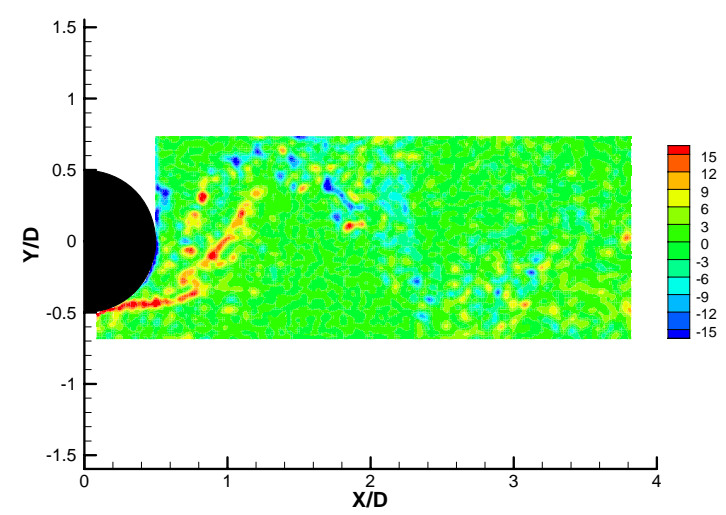

c) $\mathrm{L} / \mathrm{D}=3.70$

Figure 22. Instantaneous spanwise vorticity. 


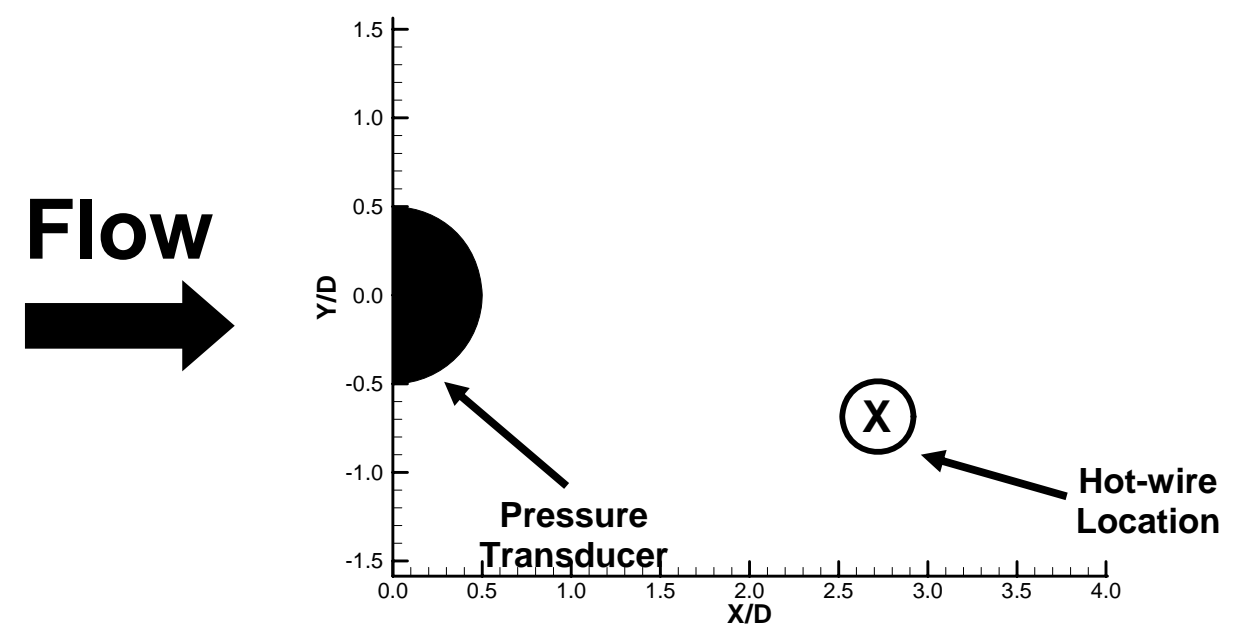

Figure 23. Pressure and velocity spectra measurement locations.

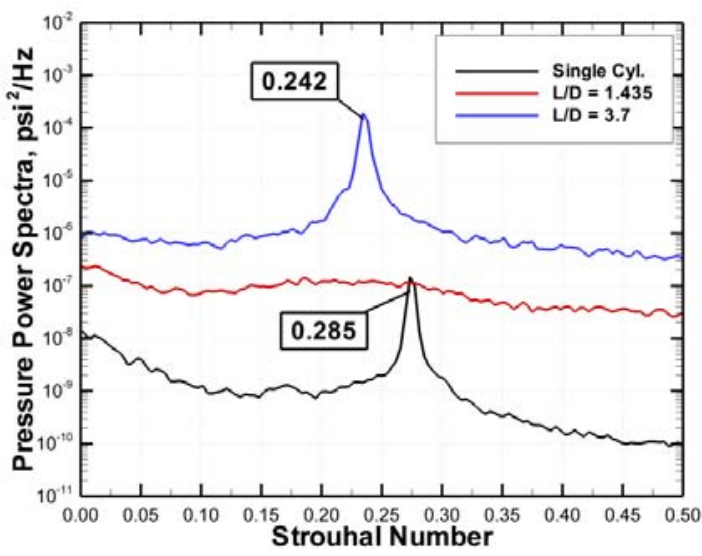

Figure 24. Pressure spectra on rear cylinder at $\theta=225 \mathrm{deg}$.

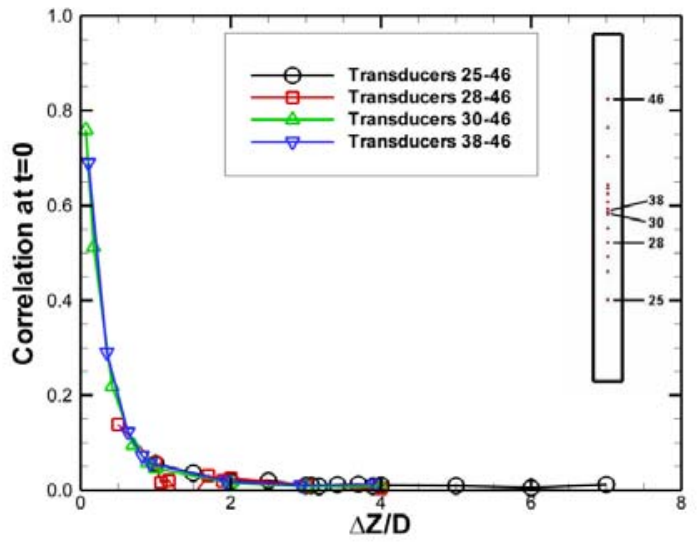

Figure 26. Fluctuating pressure correlation on rear cylinder $(\mathrm{L} / \mathrm{D}=1.435)$

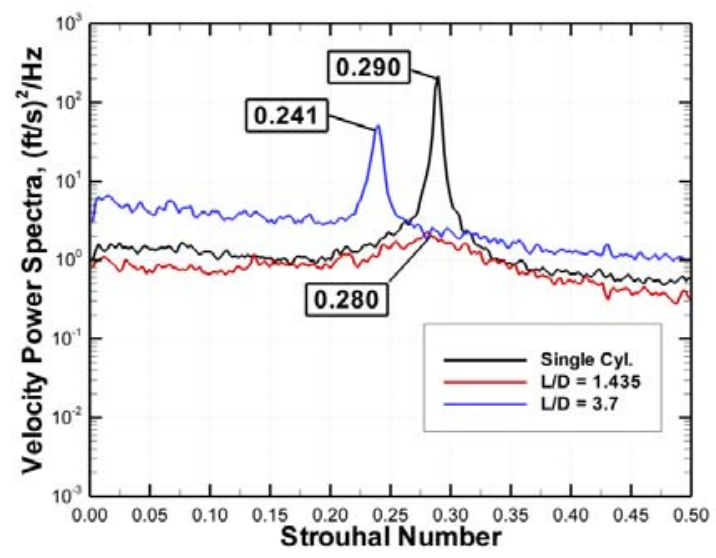

Figure 25. Velocity spectra behind rear cylinder at $\mathrm{X} / \mathrm{D}=\mathbf{2 . 7 4}, \mathrm{Y} / \mathrm{D}=\mathbf{- 0 . 6 8 5}$

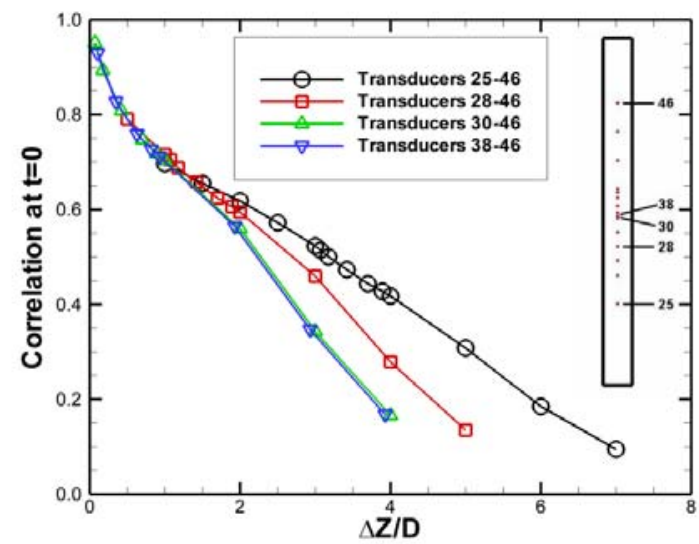

Figure 27. Fluctuating pressure correlation on rear cylinder $(\mathrm{L} / \mathrm{D}=3.7)$ 


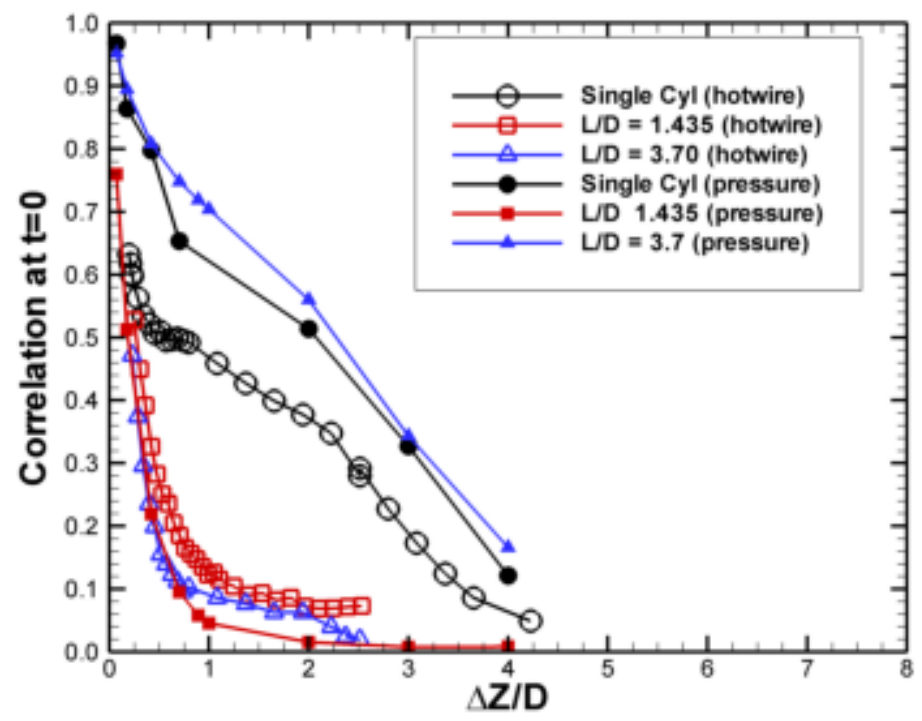

Figure 28. Correlations computed from hot-wire and surface pressure measurements. 